\title{
Personalized Medicine in Diffuse Large B-Cell Lymphoma
}

Diffuse large B-cell lymphoma (DLBCL) forms about $40 \%$ of non-Hodgkin's lymphomas in clinical practice; though it is curable with chemoimmunotherapy, those who fail R-CHOP have a dismal outcome. It is a heterogeneous disease in terms of biology and prognosis, with 3-year progression-free survival (PFS) of activated B-cell (ABC) versus germinal centre B-cell (GCB) type being $40 \%$ versus $75 \%$.

Dr. Alizadeh et al. first threw light upon the molecular subtypes via their LLMP Project where they brought out the biological and prognostic differences among DLBCL subtypes; their work was published in Nature in January 2000.

Randomized studies utilizing the biological and molecular differences between the two subtypes have thereafter been done and reported PHOENIX (ibrutinib \pm R-CHOP), ROBUST (lenalidomide \pm R-CHOP), REMoDL-B (bortezomib \pm R-CHOP), GOYA (R-CHOP vs. G-CHOP, obinutuzumab), and CALGB/ALLIANCE 50303 (R-CHOP vs. DA-EPOCH R), but none of these found a survival advantage of any of these strategies when tested without regard to specific molecular alterations, except a subset analysis of PHOENIX in patients aged $<60$ years. These studies undermined the very principle of personalized medicine, which looks at each patient as a distinct entity. In fact, it may be extremely difficult to elucidate the virtues of personalized precision medicine in a randomized manner unless only a specific molecular alteration is evaluated one at a time, instead of a large subgroup with so much heterogeneity within itself.

We look at some of the important molecular alterations in DLBCL and their utility in personalized medicine which may alter the treatment landscape of DLBCL.

\section{Ibrutinib and B-cell receptor (BCR) Signaling}

Wilson et al. from NCI Bethesda were the earliest to record responses to BTK inhibitor (BTKi) ibrutinib in refractory DLBCL. ABC tumors with BCR mutations had a higher response rate (5/9) particularly the ones with concomitant myeloid differentiation primary response 88 (MYD88) mutations (4/5), although responses did occur in those without such mutations in the $\mathrm{ABC}$ subtype, suggesting possible coexisting nongenetic mechanisms.

PHOENIX studied 838 patients with $75.9 \%$ being $\mathrm{ABC}$ type. In patients younger than 60 years, it suggested improved event-free survival (EFS), PFS, and overall survival (OS) with slightly increased serious adverse events $(35.7 \%$ vs. $28.6 \%)$ with hazards strongly favoring IR-CHOP in patients aged $<60$ years $(0.57,0.55$, and 0.33 , respectively). The 3-year PFS in ABC type was $80.5 \%$ versus $64.5 \%$ and 3 -year OS was $92.8 \%$ versus $80.9 \%$ in this cohort, suggesting a $16 \%$ advantage in PFS and $11.9 \%$ advantage in OS in absolute terms.

In patients aged $>60$ years, the adverse effects were $63.4 \%$ versus $38.2 \%$ negating any benefit of the drug in this cohort, in fact, leading to an inferior survival, and this would surely serve as a deterrent in utilizing this strategy for older patients. Overall, PHOENIX was a negative trial and the data from post hoc analysis will need to be interpreted with caution.

CD 79A/79B mutations are prevalent in about $10 \%$ and MYD88 mutations in $>30 \%$ of $\mathrm{ABC}$ type, which are druggable with $\mathrm{BTK}$ inhibition, while CARD11 mutations occurring in $10 \%$ of patients with $\mathrm{ABC}$ confer resistance to BTKi.

A multi-institutional outcome analysis by Winter et al. of $\mathrm{R} / \mathrm{R}$ DLBCL treated with ibrutinib failed to show benefit, substantiating the pitfalls of using HANS algorithm and immunohistochemistry (IHC) for designating cell of origin $(\mathrm{COO})$.

A noteworthy point is regarding RICHTER transformation wherein most patients have the $\mathrm{ABC}$ subtype clonally related to underlying chronic lymphocytic leukemia. These patients can potentially be good candidates for BCR targeting. Both ibrutinib and idelalisib have shown activity in this setting.

Idelalisib is PI3Kdelta inhibitor selective to p110 delta. CXCR4 upregulation, mTOR, and NF-kappa B activation may cause resistance to idelalisib therapy in ABC type.

Overall, IHC is a misleading strategy for $\mathrm{COO}$ if at all personalized therapy is to be employed. We may be better of using comprehensive genomic analysis rather than firing in the dark if at all we wish to utilize these targeted therapies.

\section{Role of Lenalidomide}

ROBUST could not prove usefulness of lenalidomide in $A B C$ type though the subgroups were imprecisely defined in the trial. Still, a trend toward an improved survival was visible in ipilimumab $>3$ and advanced stage patients.

$\mathrm{R}^{2}$-CHOP was tested by Nowakowski et al. at $25 \mathrm{mg}$ D1-10 with R-CHOP-21 with aspirin prophylaxis in both IHC- and GEP-defined ABC type. Although the numbers were small, the 2-year EFS was $69 \%$ in ABC DLBCL when treated with $\mathrm{R}^{2}$-CHOP which was at par with those with GCB type. They showed that lenalidomide could probably negate the adverse prognosis of $\mathrm{ABC}$ subtype. 


\section{Will Obinutuzumab Ever be Utilized?}

In an exploratory analysis of GOYA trial, a follicular lymphoma such as subtype of DLBCL defined by PTEN loss (PI3K/AKT activated), BCL-2 translocations, and a linear predictor score utilizing a nanostring test and FoundationOne Heme panel was defined.

These patients referred to as "strong GCB" patients comprised $43 \%(233 / 540)$ evaluable patients in GOYA trial.

The study group found a striking and highly significant survival advantage of G-CHOP in "strong GCB" patients, (PFS, $88 \%$ vs. $66 \%, P=0.0007$ and OS, 92\% vs. $79 \%$, $P=0.19$ ). These results reiterate the fact that personalized medicine in DLBCL would need a more thorough and prudent utilization of available analytical platforms.

\section{BCL-2 Inhibition}

About 50\% patients with DLBCL overexpress BCL-2 and $40 \%$ harbor BCL-2 translocations.

The phase 2 CAVALLI trial looked at double expressors and double-hit DLBCLs in particular based on IHC- and FISH-based algorithm. The study team utilized Venetoclax at $800 \mathrm{mg}$ once a day 4-10 in the first cycle and days $1-10$ in the cycle 2-6 with R-CHOP. Although this was a single-arm trial, patients had an impressive CR rate of $70 \%$ including those with double hit.

All in all, personalized medicine may hold the key in treating a subset of DLBCL with poor risk features and unfavorable molecular profile. Although it will be difficult to prove the utility of precision medicine in a randomized manner, due to the vast heterogeneity within the COO-defined subgroups, personalized therapy alongside immunotherapeutic and cellular strategies may improve outcomes for those destined to do poorly with conventional therapies.

Financial support and sponsorship

Nil.

\section{Conflicts of interest}

There are no conflicts of interest.

\section{Prashant Mehta1, Rahul Bhargava ${ }^{2}$}

${ }^{I}$ Department of Medical Oncology, Hematology and BMT, Asian Institute of Medical Sciences, Faridabad, ${ }^{2}$ Department of Hematology, Institute of Blood Disorders, Fortis Memorial Research Institute, Gurgaon,

Haryana, India

Address for correspondence: Dr. Prashant Mehta, Department of Medical Oncology, Hematology and BMT, Asian Institute of Medical Sciences, Faridabad, Haryana, India. E-mail: prashantcipher7@gmail.com

Submitted: $15-$ Oct-2019

Revised: 19-Dec-2019

Accepted: 12-Jan-2020

Published: 17-Feb-2020

This is an open access journal, and articles are distributed under the terms of the Creative Commons Attribution-NonCommercial-ShareAlike 4.0 License, which allows others to remix, tweak, and build upon the work non-commercially, as long as appropriate credit is given and the new creations are licensed under the identical terms.

\begin{tabular}{|l|l|}
\hline \multicolumn{2}{|c|}{ Access this article online } \\
\hline Quick Response Code: & Website: \\
& www.ijmpo.org \\
\cline { 2 - 2 } & Dol: \\
\hline
\end{tabular}

How to cite this article: Mehta $P$, Bhargava R. Personalized medicine in diffuse large B-cell lymphoma. Indian J Med Paediatr Oncol 2019;40:463-4. 